\title{
Vorwort zur 1. Auflage
}

Das vorliegende Arbeitsbuch wendet sich an Leser, die sich umfassend und grundlegend mit den Fragen der Betriebswirtschaftlichen Steuerlehre auseinandersetzen wollen, seien es Lehrende und Studierende an Universitäten, Fachhochschulen, Berufsakademien, Verwaltungsund Wirtschaftsakademien und ähnlichen Einrichtungen, seien es interessierte Praktiker.

Die Betriebswirtschaftliche Steuerlehre steht unter dem dominierenden Einfluss zahlreicher steuer- und handelsrechtlicher Vorschriften, die Auswirkungen auf fast alle unternehmerischen Bereiche und Entscheidungen entfalten. Sie stellt einen Teil der Allgemeinen Betriebswirtschaftslehre dar, in dem betriebswirtschaftliche Fragen unter spezifischen steuerlichen Aspekten behandelt werden.

Im ersten Teil („Rechnungswesen und Unternehmensbesteuerung“) wird nach einer „Einführung“ (1. Abschnitt) im 2. Abschnitt „Externes Rechnungswesen und Unternehmensbesteuerung“ auf die Gewinn- und Vermögensermittlung sowie dadurch induzierte rechnungspolitische Sachverhalte eingegangen; der 3. Abschnitt „Internes Rechnungswesen und Unternehmensbesteuerung“ befasst sich mit dem Einfluss der Besteuerung auf Investitionsentscheidungen, auf kurz- und mittelfristige Entscheidungen sowie auf Finanzierungsentscheidungen.

Der zweite Teil (,Steuerarten und Unternehmensbesteuerung“) beinhaltet einen 1. Abschnitt, der selbsterklärend überschrieben ist mit „Steuerrechtliche Grundlagen und Überblick über das deutsche Steuersystem“. Im 2. Abschnitt „Systematische Darstellung der wichtigsten Steuerarten" wird eingehend Bezug genommen auf die rechtlich determinierten Regelungen bei den wichtigsten Steuerarten. Der 3. Abschnitt „Der Einfluss der Besteuerung auf unternehmerische Entscheidungen“ zeigt ausgehend von einem Steuerbelastungsbeispiel die Einflussfaktoren der Steuerbelastung auf.

Der 1. Abschnitt „Rechtsformwahl und Rechtsformbesteuerung“ des dritten Teils („Unternehmensstruktur und Unternehmensbesteuerung“") widmet sich allen Phasen eines Unternehmens in Abhängigkeit von der Rechtsform. Nach dem selbsterklärenden Titel des 2. Abschnitts „Wahl der Zusammenschlussform und Rechtsstrukturbesteuerung“ erfolgt eine Würdigung des Umwandlungssteuergesetzes und anderer Regelungen im Zusammenhang mit der Änderung der Unternehmensstruktur im 3. Abschnitt (,Wechsel der Rechtsform und der Rechtsstruktur und ihre Besteuerung“). Im 4. Abschnitt „Standortwahl und Standortbesteuerung“ findet nach einer knappen Aufbereitung der nationalen standortabhängigen Steuerwirkungen eine ausführliche Würdigung der internationalen Standortwahl statt. Die Überlegungen dieses Abschnitts sind die Grundlage für die abschließenden Ausführungen im 5. Abschnitt (,Funktionelle Entscheidungen mit langfristiger (struktureller) Wirkung und Besteuerungswirkungen").

Die Bezeichnung „Arbeitsbuch“ habe ich aus verschiedenen Gründen bewusst gewählt:

- Das Werk soll zwar die gesamte Betriebswirtschaftliche Steuerlehre abbilden, kann und will aber kein Ersatz für komplette Lehrbücher sein; statt dessen will es in kompakter und stichwortartiger Form die relevanten Sachverhalte dieses Faches wiedergeben und insofern Grundlage für eine Erarbeitung des Stoffes im Rahmen von Lehrveranstaltungen, aber auch im Selbststudium sein. Während im ersten Fall die Lehrveranstaltung als zwei- 
te Arbeitsgrundlage dient, erfüllen diese Aufgabe im zweiten Fall die weiterführenden Literaturhinweise. Der Übersichtlichkeit halber wurden Angaben zu grundlegender Literatur in Blockzitation unter die jeweilige Hauptüberschrift gestellt. Vertiefende bzw. weiterführende Literatur lässt sich dagegen in Fußnoten finden.

- Das Buch stellt das Ergebnis langjähriger Arbeiten im Bereich der „Betriebswirtschaftlichen Steuerlehre“ dar. Die entsprechenden Skripten, die in einem guten Jahrzehnt als Hochschullehrer der Betriebswirtschaftlichen Steuerlehre, v.a. aber in den letzten fast 5 Jahren an meinem Lehrstuhl an der Universität des Saarlandes entstanden sind und immer wieder verfeinert, verbessert und aktualisiert wurden, sind hier zusammengeführt und auf den Rechtsstand des Jahres 1997 angepasst worden. Das letzte Steuerreformgesetz, das in den allerletzten Korrekturrunden Berücksichtigung finden musste, ist jenes vom 29.10.1997; seine Änderungen beziehen sich z.T. auf das Jahr 1998, aber auch teilweise rückwirkend auf den Zeitraum ab August 1997 bzw. auf das gesamte Jahr 1997. Diese - vorsichtig ausgedrückt - eigenartige Rückwirkungspraxis macht es selbst Anfang Dezember eines Jahres offenbar unmöglich, den Rechtsstand desselben Jahres wiederzugeben, könnte doch noch bis Jahresende rückwirkend eine umfassende Änderung mit Wirkung für den Anfang desselben Jahres erfolgen. Die Änderungen der „OktoberSteuergesetze“ sind im Anschluss an die „Vor-Oktober-Regelungen“ im Rahmen eigener Absätze an den betreffenden Stellen berücksichtigt worden; potenzielle spätere „Geniestreiche“ des Gesetzgebers mit Wirkung für 1997 sind nicht mehr erfasst worden, allerdings auch fast ausgeschlossen. Insofern ist der Rechtsstand 1997 (Ende des Jahres) Stand der Ausführungen.

- Ziel der Arbeit ist es, eine Lücke im Bereich der Betriebswirtschaftlichen Steuerlehre zu schließen. Auf der einen Seite wird eine - wenn auch knappe - Darstellung des gesamten Stoffes angestrebt; in ständig aktualisierter Form kann dies angesichts der Änderungsgeschwindigkeit des Steuerrechts ein umfassendes Lehrwerk kaum noch leisten. Auf der anderen Seite wird der Bezug zu einem konkreten Steuerrechtsstand - hier so gut es geht, jener des Jahres 1997 - hergestellt, so dass auch in einem Werk mit überschaubarem Umfang Detailbetrachtungen möglich sind. Anders ausgedrückt, wird der Versuch unternommen, die Breite des Faches mit Detailtreue und -tiefe sowie mit großer Aktualität zu verbinden. Dafür ist zweierlei erforderlich: erstens der Verzicht auf umfassende Ausformulierungen an allen Stellen und zweitens der Mut zur Publizierung einer Arbeit, die angesichts der Komplexität des Stoffes fast unvollkommen bleiben muss. Zur Realisierung der erforderlichen kontinuierlichen Weiterarbeit ist der Autor den Lesern für kritische Anregungen sowie für jeden Verbesserungsvorschlag dankbar.

Ohne die Unterstützung ehemaliger und aktueller wissenschaftlicher Mitarbeiterinnen und Mitarbeiter in Form zahlreicher Hinweise, Hilfen und Verbesserungsvorschläge wären meine Skripten und Fallstudien nicht möglich geworden. Bei der konkreten Fertigstellung dieses Projektes waren koordinierend und Korrektur lesend Herr Dipl.-Wirtsch.-Ing. BERND LEIDERER sowie formatierend, formalisierend und Korrektur lesend Herr Dipl.-Kfm. ANDY JUNKER tätig. Ihnen gilt mein besonderer Dank genauso wie den redaktionell und inhaltlich - durch vielfältige Hinweise und Verbesserungsvorschläge bei der Durchsicht des Manuskriptes einzelnen Passagen zugeordneten Personen; der erste Teil wurde von Frau Dipl.-Kffr. MAR- 
TINA DrEgER, der zweite Teil von Herrn Dipl.-Kfm. FrIEDBERT MAIER, die ersten drei Abschnitte des dritten Teils von Herrn Dipl.-Kfm. ANDY JUNKER und die beiden letzten Abschnitte des dritten Teils von Frau Dipl.-Kffr. MIRIAM GLESSNER begleitet. Für die Umsicht und Sorgfalt beim Schreiben von Manuskripten bin ich meiner Sekretärin, Frau DoRIS SCHNEIDER, zu Dank verpflichtet. Selbstverständlich gehen zutage tretende Unzulänglichkeiten ausschließlich zu Lasten des Autors, der dann an diesem Arbeitsbuch besonders intensiv weiterarbeiten muss. 
